\title{
Prótesis valvular aórtica percutánea: ¿Qué debemos saber?
}

\author{
Alejandro Paredes ${ }^{1}$, Alejandro Martínez. \\ 1. Residente de Cardiología \\ División de Enfermedades Cardiovasculares, Escuela de Medicina, Pontificia Universidad \\ Católica de Chile.
}

\section{Introducción:}

La estenosis aórtica (EA) es actualmente una de las enfermedades valvulares más comunes en los países desarrollados ${ }^{1}$. Su prevalencia aumenta con la edad y se diagnostica en el $4,6 \%$ de los adultos mayores de 75 años. Tiene un curso insidioso, con un largo período de latencia; sin embargo, presenta una rápida progresión posterior al inicio de los síntomas. Sin tratamiento específico, se ha descrito una mortalidad promedio de $50 \%$ a 2 años ${ }^{2}$.

El reemplazo valvular aórtico quirúrgico (RVAo) es el pilar del tratamiento de la estenosis aórtica avanzada, mejora los síntomas y aumenta la sobrevida. Sin embargo, existe un $30 \%$ de los pacientes con EA sintomática que por su riesgo no son "buenos" candidatos quirúrgicos.

Aunque globalmente la mortalidad del RVAo se reporta por debajo del 3\%, el riesgo de mortalidad y morbilidad aumentan significativamente cuando la estenosis aórtica se presenta en pacientes de edad avanzada con patologías concomitantes. Precisamente en estos casos riesgosos los clínicos han sido reacios para indicar la cirugía.

Como alternativa, en el año 2002 se implantó por primera vez una prótesis aórtica por vía percutánea (TAVI: transcatheter aortic valve implantation) $\left(^{3}\right)$. Desde entonces, la seguridad y eficacia de este nuevo tratamiento se ha confirmado tanto con los resultados de múltiples registros multicéntricos, como también con estudios aleatorizados. Así, se ha establecido con los sistemas Sapien de Edwards y CoreValve de Medtronic, una tasa de éxito $>90 \%$ y una mortalidad a los 30 días del procedimiento $<10 \%$ en la mayoría de las series. Por su parte, en forma aleatorizada, el ensayo PARTNER confirmó tanto la superioridad de la TAVI sobre el tratamiento médico en pacientes no considerados aptos para el recambio valvular quirúrgico estándar ${ }^{4}$, como la no inferioridad de la TAVI comparada con el tratamiento quirúrgico en pacientes de alto riesgo ${ }^{5}$. De este modo se dispone en la actualidad de esta nueva alternativa de tratamiento en pacientes de alto riesgo quirúrgico $\mathrm{y}$ se discute su potencial indicación en un espectro más amplio de pacientes.

Correspondencia:

Dr. Alejandro Martínez S.

División de Enfermedades Cardiovasculares

Escuela de Medicina

Pontificia Universidad Católica de Chile

E-mail: amartine@med.puc.cl 


\section{Válvulas protésicas transcatéter}

A la fecha, existen dos tipos de endoprótesis percutáneas, las cuales han sido ampliamente utilizadas en diferentes escenarios clínicos (Figura 1):

\section{Figura 1: Prótesis Sapien (A) y Core Valve (B)}

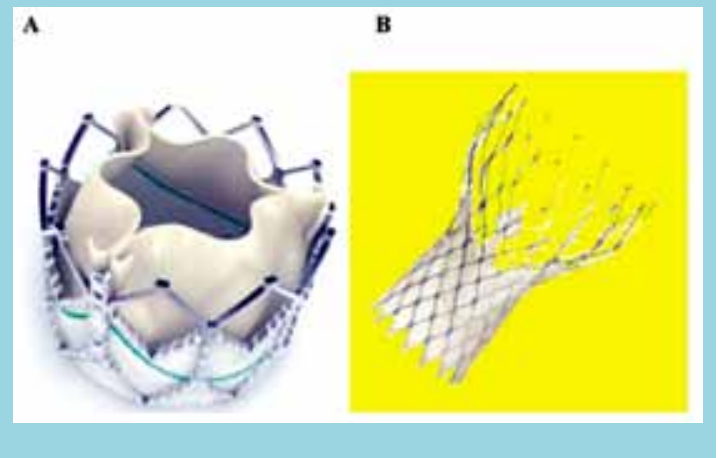

-Balón expansibles: Sapien valve ${ }^{\circledR}$, Edwards.

-Auto expansibles: CoreValve ${ }^{\circledR}$, Medtronic.

Ambas están constituidas por material biológico y se implantan por dentro de la válvula aórtica estenótica. Habitualmente, el implante es precedido por la dilatación de la válvula nativa con balón y posteriormente, tanto la expansible con balón como la auto expansible, son ubicadas e implantadas en el sitio correspondiente, usando como guía la ecocardiografía y la radioscopía.

Ver tabla $\mathrm{N}^{\circ} 1$

\begin{tabular}{|c|c|c|}
\hline & $\begin{array}{c}\text { Edwards Sapien } \\
\text { XT }\end{array}$ & Medtronic CoreValve \\
\hline Generacion de válvula & Tercera & Tercera \\
\hline Marco & Cromo cobalto & Nitinol \\
\hline Velos & Pericardio bovino & Pericardio porcino \\
\hline Mecanismo de expansión & Balón expandible & Auto expandible \\
\hline Balonplastia previa requerida & $\mathrm{Si}$ & $\mathrm{Si}$ \\
\hline Reposicionamiento de prótesis & No & Previo a la liberación \\
\hline Fijación anular/valvular & $\mathrm{Si}$ & $\mathrm{Si}$ \\
\hline Diámetros & $23,26 \mathrm{~mm}$ & $26,29 \mathrm{~mm}$ \\
\hline Diámetros recomendado del anillo & $18-25 \mathrm{~mm}$ & $20-27 \mathrm{~mm}$ \\
\hline Largo & $15-17 \mathrm{~mm}$ & $53-55 \mathrm{~mm}$ \\
\hline Diámetro del sistema de liberacion & $18 F \& 19 F$ & $18 \mathrm{~F}$ \\
\hline Diámetro externo de la vaina & $7.3 \mathrm{~mm}$ & $7.3 \mathrm{~mm}$ \\
\hline Diámetro arterial mínimo & $6 \mathrm{~mm}$ & $6 \mathrm{~mm}$ \\
\hline Útil en estenosis aórtica & $\mathrm{Si}$ & $\mathrm{Si}$ \\
\hline Útil en insuficiencia aórtica & Si es comb. con estenosis & $\mathrm{Si}$ \\
\hline Útil a nivel pulmonar & $\mathrm{Si}$ & No \\
\hline Valve in Valve & En 4 posiciones valvulares & Sólo aórtica \\
\hline Acceso transfemoral & $\mathrm{Si}$ & $\mathrm{Si}$ \\
\hline Acceso transapical & $\mathrm{Si}$ & No \\
\hline Acceso transaxilar & $\mathrm{Si}$, aunque experiencia limitada & $\mathrm{Si}$ \\
\hline Seguimiento a largo plazo reportado & 5 años & 3 años \\
\hline Marcapasos definitivo & $4-8 \%$ & $15-40 \%$ \\
\hline
\end{tabular}

\section{Accesos utilizados y/o técnicas de implante}

Para acceder al sitio de implante por vía endovascular, es necesario avanzar desde una arteria periférica los catéteres que contienen la válvula protésica. Estos catéteres todavía son de gran tamaño. Su diámetro, dependiendo del tipo de válvula, fluctúa entre 6 y $8 \mathrm{~mm}$ (18-24F). Por esta razón es necesario evaluar muy bien la vía de acceso a utilizar.

\section{Vía femoral:}

Es considerada la primera opción en la mayoría de los centros que realizan TAVI, siendo respaldada por una gran cantidad de evidencia. Se puede utilizar mediante exposición quirúrgica o también, cuando se dan las condiciones anatómicas, como una técnica enteramente percutánea, con punción arterial y uso de dispositivos especiales para su cierre.

\section{Vía subclavia:}

Es una alternativa al acceso transfemoral cuando éste no está disponible. La arteria subclavia, en general, mide menos que las ilíacas. Alcanza en promedio entre 5 a 7 mm. Se puede utilizar para el implante de la prótesis auto expansible Core Valve, que tiene menor diámetro ${ }^{9}$ Requiere una exposición quirúrgica, mediante incisión infraclavicular. En el sitio de entrada se anastomosa un tubo protésico que permite ser exteriorizado, para avanzar los catéteres por su interior. Alcanzada la aorta ascendente, la técnica de implante es igual a la femoral. Exepcionalmente se ha usado la arteria axilar $\left({ }^{10}\right)$.

\section{Transapical:}

Ha sido reportada como la primera alternativa al acceso transfemoral ${ }^{6}$. Requiere una toracotomía lateral izquierda y una punción directa a nivel del ápex del ventrículo izquierdo. Desde esta ventana se introduce el catéter liberador y se procede al implante.

Entre las potenciales ventajas lo principal es que se evita el avance de grandes catéteres a través del sistema ileofemoral, arco aórtico y aorta ascendente, con la consiguiente disminución de riesgos vasculares. Además, puede permitir un mejor alineamiento técnico al momento del implante valvular, sobre todo en aquellos pacientes con aortas con disposición horizontal.

Sin embargo, la complejidad del proceso, necesidad de toracotomía, posibilidad de injuria miocárdica y los riesgos asociados (ej: sangrados e insuficiencia mitral), han limitado su uso ${ }^{7}$.

\section{Vía transaórtica:}

Se ha introducido recientemente como acceso alternativo para ambos dispositivos valvulares. A pesar de requerir una pequeña esternotomía, podría ser elegible cuando no se puede usar la vía femoral o subclavia ${ }^{8}$. 


\section{Indicaciones de TAVI}

En la actualidad, debido a sus limitaciones y potenciales complicaciones, se indica en pacientes portadores de EA avanzada sintomática con muy alto riesgo para la cirugía convencional ${ }^{11}$, cuando cumplen con los criterios anatómicos que se describirán.

La gran mayoría de los pacientes que hasta el momento han recibido este tratamiento son octogenarios con un riesgo estimado de mortalidad operatoria $>20 \%$. Además, se ha indicado en los pacientes con menos comorbilidad, pero que presentan dificultades anatómicas para la cirugía, como por ejemplo, aorta "de porcelana".

\section{Estimación del riesgo quirúrgico}

Para establecer la indicación de TAVI, es importante la estimación precisa del riesgo quirúrgico en estos pacientes. Para esto se dispone de variadas calculadoras de riesgo, que pueden ser subjetivas e imprecisas, y no incluir características clínicas que pueden impactar en la mortalidad.

Entre ellas destacan el modelo de la Society of Thoracic Surgeons (STS), EuroSCORE, Ambler risk score, entre otros.

Recientemente, se demostró la superioridad del STS score sobre el EuroSCORE en la predicción de mortalidad en pacientes de alto riesgo ${ }^{12}$.

En la práctica habitual, se evalúan estos pacientes en un equipo médico-quirúrgico que contempla los resultados de estas mediciones y todas las otras características clínicas.

\section{Evaluación imagenológica pre-procedimiento}

Como se mencionó, es muy importante evaluar la anatomía de los sitios de acceso y de la aorta, para establecer la seguridad del avance de estos grandes catéteres, pero también, se necesita precisar las características de la raíz aórtica, donde se implantará la prótesis.

En este sentido importan preferentemente la estructura de la válvula, el diámetro del anillo, la amplitud de los senos de valsalva, la distancia de la base de los velos valvulares al origen de las coronarias, las características del tracto de salida del ventrículo izquierdo y el diámetro de la aorta ascendente proximal.

Desde el punto de vista de la válvula, los resultados son menos favorables en las bicúspides y en las que tienen calcificación asimétrica. El diámetro del anillo valvular define el tamaño de la prótesis. Las otras mediciones en la raíz de la aorta permiten estimar el riesgo de obstrucción coronaria y la calidad de la aposición y fijación del dispositivo.

Para hacer las evaluaciones vasculares se utilizan la angiografía invasiva y el angioTAC. Ambas permiten una estimación muy adecuada de la aorta y del territo- rio arterial periférico. Para la determinación exacta de la estructura de la válvula, ayudan los métodos angiográficos, pero la ecocardiografía transesofágica permite una evaluación más completa. Lamentablemente la determinación del tamaño del anillo no es perfecta con este método. El anillo no es exactamente circular, por lo que se puede subestimar su diámetro. Su reconstrucción tridimensional, sea con ecografía 3D o con el angioTAC, permite una medición del perímetro, y así obtener el diámetro promedio, que probablemente sea el más cercano al resultado óptimo ${ }^{13}$.

En la práctica, para obtener estas mediciones, se utilizan los 3 métodos mencionados. Se requieren estudios adicionales a futuro que ayuden a dilucidar cuál es el gold estándar en lo que respecta al mejor método imagenológico de evaluación previo a la TAVI.

Paralelamente, en la evaluación previa al procedimiento, se realiza coronariografía. Permite descartar a los pacientes con lesiones ostiales y definir la necesidad de angioplastía previa, en casos con estenosis avanzada en los vasos coronarios principales.

\section{Mortalidad}

Los resultados de las diversas series publicadas se han resumido en la tabla $\mathrm{N}^{\circ} 2$

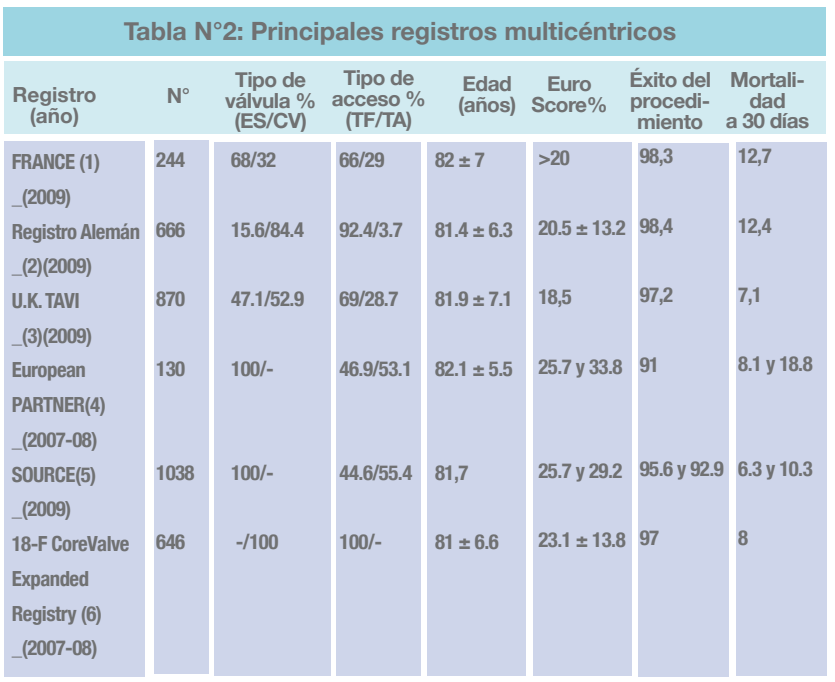

ES: Edwards system; CV: CoreValve; TF: Transfemoral; TA: Transapical.

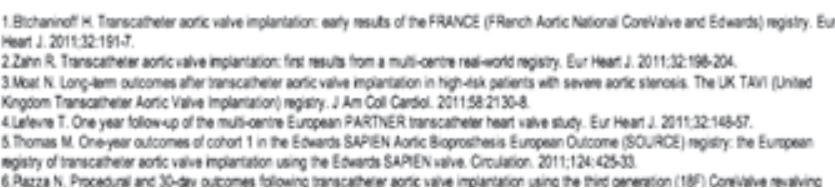

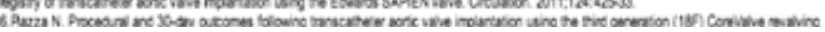

\section{Complicaciones del procedimiento}

Esta definición no ha sido del todo estandarizada, lo cual podría explicar algunas variaciones en las tasas 
reportadas en los diferentes estudios. El año 2011, la VARC (Valve Academic Research Consortium) propuso un consenso de definiciones al respecto. ${ }^{14}$

Este documento representa un importante paso hacia la unificación de conceptos en lo que respecta a las endoprótesis percutáneas.

\section{1.- Complicaciones vasculares mayores}

El uso de introductores y catéteres de gran tamaño (18-24 French) en pacientes mayores, ha llevado a altas tasas de complicaciones vasculares en relación al procedimiento (5-10\%)..$^{15}$

Una evaluación correcta de las arterias ileofemorales y el uso de alternativas a la vía transfemoral, en los casos en que este acceso es menos favorable, ha llevado a una disminución de estos riesgos. ${ }^{16}$ La ocurrencia de estas complicaciones mayores ha demostrado ser un predictor independiente de mortalidad a 30 días. ${ }^{17} \mathrm{Ac}$ tualmente, la mayoría de los centros usan dispositivos de cierre percutáneo en los casos abordados vía transfemoral. ${ }^{18}$

\section{2.- Accidentes cerebrovasculares (ACV)}

La ocurrencia de eventos cerebrovasculares es una de las complicaciones más temidas. La tasa dentro de los 30 primeros días es de alrededor de $3.5 \%$, según lo reportado en el estudio PARTNER. ${ }^{19,20}$

Dentro de este estudio, la cohorte no operable también mostró tasas más altas de ACV o accidentes isquémicos transitorios a 30 días $(6.7 \% \mathrm{v} / \mathrm{s} 1.7 \%, \mathrm{p}=0.03)$ y a un año de seguimiento $(10.6 \% \mathrm{v} / \mathrm{s} 4.5 \%, \mathrm{p}=0.04)$ entre aquellos pacientes asignados a TAVI comparados con aquellos manejados de manera conservadora (incluyendo balonplastía aórtica en el $84 \%$ de los casos). ${ }^{21}$

Estudios con RNM cerebral con difusión han mostrado una incidencia de isquemia cerebral silente tan alta como $66-84 \%$ posterior al implante de la TAVI ${ }^{22,23}$, independiente del tipo de válvula utilizada y el abordaje ${ }^{24}$. Por lo tanto, sería más frecuente que los episodios de ACV detectados habitualmente.

Aunque los estudios con Doppler transcraneal han mostrado que la embolía cerebral puede ocurrir en cualquier momento durante el implante del dispositivo, la mayoría se produce durante el posicionamiento e implantación de la válvula protésica ${ }^{25}$, indicando que la embolización de partículas proveniente de la válvula nativa calcificada podría ser un importante mecanismo de embolías cerebrales asociados a TAVI. Esto podría explicar el porqué varios estudios han fallado en demostrar la relevancia en este sentido en lo que respecta a la vía de abordaje. Actualmente, se encuentran en desarrollo de dispositivos de protección frente a estas partículas embólicas. ${ }^{26}$ Un subestudio del PARTNER propuso que los eventos cerebrovasculares ocurridos precozmente posterior al implante de la TAVI se asociaron a áreas valvulares aórticas nativas más pequeñas; y aquellos ocurridos tardíamente estaban principalmente relacionados a carga aterosclerótica más altas. ${ }^{27}$

De manera empírica, se ha recomendado la doble antiagregación plaquetaria con aspirina y clopidogrel posterior a la TAVI, pero futuros estudios determinarán el tratamiento antitrombótico óptimo posterior a estos procedimientos. ${ }^{28}$

\section{3.- Obstrucción coronaria}

La obstrucción del ostium coronario (especialmente de la coronaria izquierda) puede ocurrir posterior al implante de la TAVI, pero la incidencia global es muy baja $(<1 \%)^{20}$

Esta complicación se encuentra asociada al desplazamiento del velo aórtico nativo hacia el ostium durante el implante, y el riesgo parece ser más alto en aquellos pacientes con velos altamente calcificados. El uso de angiografía aórtica durante la valvuloplastía con balón antes del implante de la prótesis podría ayudar a determinar si el desplazamiento del velo valvular podría comprometer el flujo coronario. ${ }^{29}$

\section{4.- Infarto del miocardio}

Se han descritos tasas variables de infarto del miocardio asociado a TAVI que van desde $0-16.3 \%{ }^{20,30}$. Esta variabilidad es probablemente relacionada a la falta de uniformidad en la definición de infarto peri procedimiento entre los distintos estudios.

La definición VARC de infarto miocárdico peri procedimiento considera la ocurrencia de nuevos síntomas o signos de isquemia asociado con una elevación de biomarcadores cardíacos (de preferencia creatinina quinasa MB) al menos de 10 veces sobre el límite normal superior o al menos 5 veces el límite superior con nuevas ondas Q patológicas en el electrocardiograma. ${ }^{14}$

Existiría mayor grado de injuria miocárdica la cual se asociaría a menor recuperación en la fracción de eyección del ventrículo izquierdo e incremento de la mortalidad en el seguimiento a mediano plazo.?

\section{5.- Daño renal agudo}

La incidencia de daño renal agudo y la necesidad de soporte renal después de la TAVI varían desde $11.7 \%$ a $28 \%$, y desde $1.4 \%$ a $15.7 \%$, respectivamente ${ }^{31-35}$.

En el estudio PARTNER, en el grupo de alto riesgo, la necesidad de diálisis fue similar en el grupo TAVI y en el grupo asignado a recambio valvular quirúrgico, ya sea a 30 días (2.9\% vs $3.0 \%$ ) como en el seguimiento a un año $(5.4 \% \mathrm{v} / \mathrm{s} 6.5 \%)^{20}$.

Debe tenerse en cuenta que la enfermedad renal crónica es una enfermedad común entre los pacientes evaluados 
para el implante de TAVI. La transfusión de hemoderivados periprocedimiento ha sido reconocida como un factor predictor mayor de daño renal agudo post-TAVI, lo que puede deberse a que el riesgo de lesión renal se asocia a la cuantía de sangramiento ${ }^{31,36}$.

\section{6.- Trastornos de la conducción}

La aparición de nuevos trastornos de la conducción intraventricular, principalmente el bloqueo de rama izquierda, ocurren frecuentemente después del procedimiento (7-18\% con prótesis balón expandible y 30-83\% con las auto expandible $)^{37-39}$. El daño mecánico directo y la inflamación de la rama izquierda del haz de His, creada por el stent conteniendo la prótesis valvular son mecanismos potenciales de estos trastornos. La presencia de un bloqueo previo de rama derecha parece ser un importante predictor de bloqueo aurículoventricular completo y la necesidad de implante de marcapaso definitivo posterior a la TAVI. ${ }^{40}$ Entre las variables, el uso de CoreValve y el implante protésico más profundo (> de $5 \mathrm{~mm}$ desde el anillo aórtico), son 2 de los determinantes más importantes en la ocurrencia de trastornos de la conducción y necesidad de marcapasos ${ }^{41-43}$. La necesidad de marcapasos permanente con CoreValve en alrededor de 20\% (9-40\% según diversas series) comparado con un 5\% en las prótesis Sapien (5-7\%) $)^{19,39,43,44}$. Estas diferencias podrían explicarse por la profundidad en el implante de la prótesis y el mayor grado de presión generada sobre el septum ventricular por el marco del dispositivo.

\section{Resultados sobre la Hemodinamia postprocedimiento}

La gran mayoría de las series publicadas reportando su experiencia con las válvulas Sapien o CoreValve han demostrado de manera sistemática excelentes resultados hemodinámicos, con gradientes residuales medios $<15$ mmHg y áreas valvulares $>1,5 \mathrm{~cm} 2$ tras la implantación de la válvula. Asimismo, se ha confirmado que estos resultados se mantienen en el seguimiento a mediano plazo. Debido a una incompleta aposición de la prótesis en el anillo valvular es posible, sin embargo, la aparición de insuficencia valvular tras el implante. Aunque puede ser causada por un error de técnica, la mayoría de las veces se debe a condiciones anatómicas de la raíz de la aorta. Con una meticulosa evaluación previa y una adecuada selección del tamaño de la prótesis, la aparición de insuficiencia aórtica avanzada es poco frecuente. De hecho, la incidencia de insuficiencia aórtica moderada y avanzada, se han reportado inferiores al 10\% (0-26\%) y al $5 \%(0-10 \%)$, respectivamente ${ }^{45}$. Cuando se presenta, de acuerdo a su mecanismo, puede tratarse con sobreimpactación con balón o con la colocación de una segunda válvula percutánea (Valve-in-valve).

\section{Pronóstico y calidad de vida}

Moat et al, en el registro UK TAVI (United Kigdom Transcatheter Aortic Valve Implantation), incluyeron de forma prospectiva los datos de 877 procedimientos realizados en 870 pacientes hasta diciembre de 2009 y consiguieron obtener los datos de mortalidad de al menos un año en todos los pacientes ${ }^{43}$.

La supervivencia a los 30 días fue del 92,9\%, a un año de $78,6 \%$ y a dos años del $73,7 \%$, con una caída marcada en el periodo comprendido entre el primer mes y el primer año. El análisis estadístico mostró que la supervivencia estuvo significativamente influenciada por la presencia de insuficiencia renal, enfermedad coronaria o un abordaje diferente al femoral. Sin embargo, los únicos factores que demostraron en el modelo multivariado ser predictores independientes fueron la presencia de disfunción ventricular avanzada, insuficiencia aórtica residual moderada/avanzada y la enfermedad pulmonar obstructiva crónica.

\section{Contraindicaciones para TAVI y accesos}

Ver tabla $\mathrm{N}^{\circ} 3$

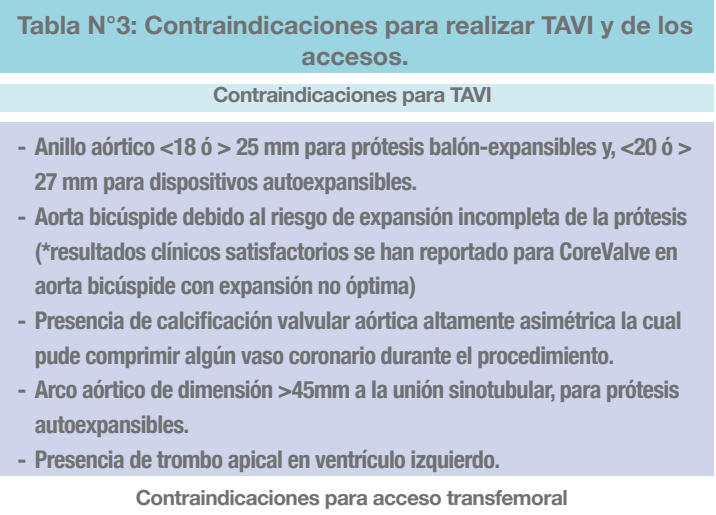

Arterias iliacas con severas calcificaciones, tortuosidad, diámetro pequeño ( $<6$ a $9 \mathrm{~mm}$ dependiendo del dispositivo) o bypass aorto-femora previo.

- Aorta con severa angulación, ateromatosis severa (arco y aorta ascendente) coartación, aneurisma de aorta abdominal con trombo mural protruyente.

-Aorta ascendente transversa para dispositivos expansibles con balón.

Contraindicaciones para acceso transapical

-Cirugía ventricular izquierda previa con uso de parche (ej: Procedimiento de Dor)

-Pericardio calcificado

-Insuficiencia respiratoria severa.9 


\section{El futuro...}

Están en evaluación clínica nuevos modelos de endoprótesis valvulares. Se trata de incorporar características para reducir el diámetro de los catéteres, facilitar el posicionamiento preciso, reducir las insuficiencias paravalvulares, o permitir la recuperación de estos dispositivos. En general, estas válvulas de próxima generación son auto-expandibles con los consiguientes beneficios antes señalados.

\section{Resumen}

En los últimos años hemos asistido al desarrollo de dispositivos para el tratamiento percutáneo de las valvulopatías. La TAVI ha sido desarrollada para tratar aquellos pacientes con estenosis aórtica avanzada sintomática con un alto riesgo quirúrgico para el reemplazo valvular. Presenta resultados clínicos superiores al tratamiento médico estándar incluyendo la valvulotomía percutánea. En comparación al RVAo quirúrgico presenta similares tasas de sobrevida a un año de seguimiento.

Nuevos estudios están siendo realizados en un intento de extender la indicación de TAVI a pacientes de menor riesgo y más jóvenes. Sin embargo, para que ello ocurra, parece ser necesario resolver algunas de las limitaciones del procedimiento, como la necesidad de marcapasos, incidencia de accidente cerebrovascular y la insuficiencia paravalvular en sus diferentes grados.

Cabe destacar finalmente, la importancia de un abordaje multidisciplinario al evaluar a este grupo de pacientes con el fin de obtener mejores resultados clínicos.

\section{Referencias:}

1. IUNG B, BARON G, BUTCHART EG, DELAHAYE F, GOHLKE-BARWOLF C, LEVANG OW, et al. A prospective survey of patients with valvular heart disease in Europe: The Euro Heart Survey on Valvular Heart Disease. European heart journal. 2003;24:1231-43.

2. NKOMO VT, GARDIN JM, SKELTON TN, GOTTDIENER JS, SCOTT CG, ENRÍQUEZ-SARANO M. Burden of valvular heart diseases: a population-based study. Lancet. 2006;368:1005-11.

3. CRIBIER A, ELTCHANINOFF H, BASH A, BORENSTEIN N, TRON C, BAUER F, et al. Percutaneous transcatheter implantation of an aortic valve prosthesis for calcific aortic stenosis: first human case description. Circulation. 2002;106:3006-8.

4. LEON MB, SMITH CR, MACK M, MILLER DC, MOSES JW, SVENSSON LG, et al. Transcatheter aortic-valve implantation for aortic stenosis in patients who cannot undergo surgery. The New England journal of medicine. 2010;363:1597-607.

5. SMITH CR, LEON MB, MACK MJ, MILLER DC, MOSES JW, SVENSSON LG, et al. Transcatheter versus surgical aorticvalve replacement in high-risk patients. The New England journal of medicine. 2011;364:2187-98.

6. BAGUR R, RODÉS-CABAU J, DOYLE D, DE LAROCHELLIÈRE R, VILLENEUVE J, LEMIEUX J, et al.Usefulness of TEE as the primary imaging technique to guide transcatheter transapical aortic valve implantation. JACC Cardiovasc Imaging. 2011;4:115-24.

7. RODÉS-CABAU J, GUTIÉRREZ M, BAGUR R, DE LAROCHELLIÈRE R, DOYLE D, CÔTÉ M, et al. Incidence, predictive factors, and prognostic value of myocardial injury following uncomplicated transcatheter aortic valve implantation. J Am Coll
Cardiol. 2011:57:1988-99.

8. LATSIOS G, GERCKENS U, GRUBE E. Transaortic transcatheter aortic valve implantation: a novel approach for the truly [ldquo]no-access option[rdquo] patients. Catheter Cardiovasc Interv. 2010;75:1129-36.

9. PETRONIO AS, DE CARLO M, BEDOGNI F, MARZOCCHI A, KLUGMANN S, MAISANO F, et al. Safety and efficacy of the subclavian approach for transcatheter aortic valve implantation with the CoreValve revalving system. Circ Cardiovasc Interv. 2010;3:359-66.

10. DE ROBERTIS F, ASGAR A, DAVIES S, DELAHUNTY N, KELLEHER A, TRIMLETT R, et al. The left axillary artery[mdash]a new approach for transcatheter aortic valve implantation. Eur J Cardiothorac Surg. 2009;36:807-12.

11. VAHANIAN A, ALFIERI O, AL-ATTAR N, ANTUNES M, BAX J, CORMIER B, et al. Transcatheter valve implantation for patients with aortic stenosis: a position statement from the European Association of Cardio-Thoracic Surgery (EACTS) and the European Society of Cardiology (ESC), in collaboration with the European Association of Percutaneous Cardiovascular Interventions (EAPCI). European heart journal. 2008;29:1463-70.

12. BEN-DOR I, GAGLIA MA JR., BARBASH IM, MALUENDA G, HAUVILLE C, GONZALEZ MA, et al. Comparison between Society of Thoracic Surgeons score and logistic EuroSCORE for predicting mortality in patients referred for transcatheter aortic valve implantation. Cardiovascular revascularization medicine: including molecular interventions. 2011;12:345-9.

13. MESSIKA-ZEITOUN D, SERFATY JM, BROCHET E, DUCROCQ G, LEPAGE L, DETAINT D, et al. Multimodal assessment of the aortic annulus diameter. Implications for transcatheter aortic valve implantation. J Am Coll Cardiol. 2010;55:186-94. 
14. LEON MB, PIAZZA N, NIKOLSKY E, BLACKSTONE EH, CUTLIP DE, KAPPETEIN AP, et al. Standardized endpoint definitions for transcatheter aortic valve implantation clinical trials: A consensus report from the Valve Academic Research Consortium. J Am Coll Cardiol. 2011;57:253-69.

15. HAYASHIDA K, LEFÈVRE T, CHEVALIER B, HOVASSE T, ROMANO M, GAROT P, et al. Transfemoral aortic valve implantation new criteria to predict vascular complications. JACC Cardiovasc Interv. 2011;4:851-8.

16. SAIA F, BORDONI B, MARROZZINI C, CIUCA C, MORETTI C, BRANZI A, et al. Incidence, prognostic value and management of vascular complications with transfemoral transcatheter aortic valve implantation. Future Cardiol. 2011;7:321-31.

17. TAMBURINO C,CAPODANNO D, RAMONDOA,PETRONIO AS, ETTORI F, SANTORO G, et al. Incidence and predictors of early and late mortality after transcatheter aortic valve implantation in 663 patients with severe aortic stenosis. Circulation. 2011;123:299-308

18. COCKBURN J, DE BELDER A, BROOKS M, HUTCHINSON N, HILLA, TRIVEDI U, et al. Large caliber arterial access device closure for percutaneous aortic valve interventions: use of the Prostar system in 118 cases. Catheter Cardiovasc Interv. 2012;79:143-9.

19. RODÉS-CABAU J, WEBB JG, CHEUNG A, YE J, DUMONT E, FEINDEL CM, et al. Transcatheter aortic valve implantation for the treatment of severe symptomatic aortic stenosis in patients at very high or prohibitive surgical risk. Acute and late outcomes of the multicenter Canadian experience. J Am Coll Cardiol. 2010;55:1080-90.

20. SMITH CR, LEON MB, MACK MJ, MILLER DC, MOSES JW, SVENSSON LG, et al. Transcatheter versus Surgical Aortic-Valve Replacement in High-Risk Patients. N Engl J Med. 2011;364:2187-98.

21. LEON MB, SMITH CR, MACK M, MILLER DC, MOSES JW, SVENSSON LG, et al. Transcatheter aortic-valve implantation for aortic stenosis in patients who cannot undergo surgery. N Engl J Med. 2010;363:1597-607.

22. KAHLERT P, KNIPP SC, SCHLAMANN M, THIELMANN M, AL-RASHID F, WEBER M, et al. Silent and apparent cerebral ischemia after percutaneous transfemoral aortic valve implantation: a diffusion-weighted magnetic resonance imaging study. Circulation. 2010;121:870-8.

23. GHANEM A, MÜLLER A, NÄHLE CP, KOCUREK J, WERNER N, HAMMERSTINGL C, et al. Risk and fate of cerebral embolism after transfemoral aortic valve implantation. A prospective pilot study with diffusion-weighted magnetic resonance imaging. J Am Coll Cardiol. 2010;55:1427-32.

24. RODÉS-CABAU J, DUMONT E, BOONE RH, LAROSE E, BAGUR R, GURVITCH R, et al. Cerebral embolism following transcatheter aortic valve implantation: compari- son of transfemoral and transapical approaches. J Am Coll Cardiol. 2011;57:18-28.

25. SZETO WY, AUGOUSTIDES JG, DESAI ND, MOELLER P, MCGARVEY ML, WALSH E, et al. Cerebral embolic exposure during transfemoral and transapical transcatheter aortic valve replacement. J Card Surg. 2011;26:348-54.

26. NIETLISPACH F, WIJESINGHE N, GURVITCH R, TAY E, CARPENTER JP, BURNS C, et al. An embolic deflection device for aortic valve interventions. JACC Cardiovasc Interv. 2010;3:1133-8.

27. MILLER DC, BLACKSTONE EH, MACK MJ, SVENSSON LG, KODALI SK, KAPADIA S, et al. Transcatheter (TAVR) versus surgical (AVR) aortic valve replacement: incidence, hazard, determinants, and consequences of neurological events in the PARTNER Trial.

28. PIAZZA N, GRUBE E, GERCKENS U, DEN HEIJER P, LINKE A, LUHA O, et al. Procedural and 30-day outcomes following transcatheter aortic valve implantation using the third generation (18F) CoreValve revalving system: results from the multicentre, expanded evaluation registry 1-year following CE mark approval. EuroIntervention: journal of EuroPCR in collaboration with the Working Group on Interventional Cardiology of the European Society of Cardiology. 2008;4:242-9.

29. GOGAS BD,ZACHAROULIS AA,ANTONIADIS AG. Acute coronary occlusion following TAVI. Catheter Cardiovasc Interv. 2011;77:435-8.

30. CRIBIER A, ELTCHANINOFF H, TRON C, BAUER F, AGATIELLO C, SEBAGH L, et al. Early experience with percutaneous transcatheter implantation of heart valve prosthesis for the treatment of end-stage inoperable patients with calcific aortic stenosis. J Am Coll Cardiol. 2004;43:698-703.

31. NUIS RJ, VAN MIEGHEM NM, TZIKAS A, PIAZZA N, OTTEN AM, CHENG J, et al. Frequency, determinants, and prognostic effects of acute kidney injury and red blood cell transfusion in patients undergoing transcatheter aortic valve implantation. Catheter Cardiovasc Interv. 2011;77:881-9.

32. SINNING JM, GHANEM A, STEINHÄUSER H, ADENAUER V, HAMMERSTINGL C, NICKENIG G, et al. Renal function as predictor of mortality in patients after percutaneous transcatheter aortic valve implantation. JACC Cardiovasc Interv. 2010;3:1141-9.

33. BAGUR R, WEBB JG, NIETLISPACH F, DUMONT E, DE LAROCHELLIÈRE R, DOYLE D, et al. Acute kidney injury following transcatheter aortic valve implantation: predictive factors, prognostic value, and comparison with surgical aortic valve replacement. Eur Heart J. 2010;31:865-74.

34. AREGGER F, WENAWESER P, HELLIGE GJ, KADNER A, CARREL T, WINDECKER S, et al. Risk of acute kidney injury in patients with severe aortic valve stenosis undergoing transcatheter valve replacement. Nephrol Dial Transplant. 2009;24:2175-9. 
35. ELHMIDI Y, BLEIZIFFER S, PIAZZA N, HUTTER A, OPITZ

A, HETTICH I, et al. Incidence and predictors of acute kidney injury in patients undergoing transcatheter aortic valve implantation. Am Heart J. 2011;161:735-9.

36. VAN LINDEN A, KEMPFERT J, RASTAN AJ, HOLZHEY D, BLUMENSTEIN J, SCHULER G, et al. Risk of acute kidney injury after minimally invasive transapical aortic valve implantation in 270 patients. Eur J Cardiothorac Surg. 2011;39:835-43.

37. SINHAL A, ALTWEGG L, PASUPATI S, HUMPHRIES KH, ALLARD M, MARTIN P, et al. Atrioventricular block after transcatheter balloon expandable aortic valve implantation. JACC Cardiovasc Interv. 2008;1:305-9.

38. PIAZZA N, ONUMA Y, JESSERUN E, KINT PP, MAUGENEST AM, ANDERSON RH, et al. Early and persistent intraventricular conduction abnormalities and requirements for pacemaking after percutaneous replacement of the aortic valve. JACC Cardiovasc Interv. 2008;1:310-6.

39. ERKAPIC D. Electrocardiographic and further predictors for permanent pacemaker requirement after transcatheter aortic valve implantation. Europace : European pacing, arrhythmias, and cardiac electrophysiology : journal of the working groups on cardiac pacing, arrhythmias, and cardiac cellular electrophysiology of the European Society of Cardiology. 2010;12:1188-90.

40. KHAWAJA MZ, RAJANI R, COOK A, KHAVANDI A, MOYNAGH A, CHOWDHARY S, et al. Permanent pacemaker insertion after CoreValve transcatheter aortic valve implantation: incidence and contributing factors (the UK CoreValve collaborative).
Circulation. 2011;123:951-60.

41. ELTCHANINOFF H, PRAT A, GILARD M, LEGUERRIER A, BLANCHARD D, FOURNIAL G, et al. Transcatheter aortic valve implantation: early results of the FRANCE (FRench Aortic National CoreValve and Edwards) registry. Eur Heart J. 2011;32:191-7.

42. BOSMANS JM, KEFER J, DE BRUYNE B, HERIJGERS P, DUBOIS C, LEGRAND V, et al. Procedural, 30-day and one year outcome following CoreValve or Edwards transcatheter aortic valve implantation: results of the Belgian national registry. Interact Cardiovasc Thorac Surg. 2011;12:762-7.

43. MOAT NE,LUDMAN P, DE BELDER MA, BRIDGEWATER B, CUNNINGHAM AD, YOUNG CP, et al. Long-term outcomes after transcatheter aortic valve implantation in high-risk patients with severe aortic stenosis. The UK TAVI (United Kingdom Transcatheter Aortic Valve Implantation) registry. J Am Coll Cardiol. 2011;58:2130-8.

44. ZAHN R, GERCKENS U, GRUBE E, LINKE A, SIEVERT H, EGGEBRECHT H, et al. Transcatheter aortic valve implantation: first results from a multi-centre real-world registry. Eur Heart J. 2011;32:198-204.

45. CLAVEL MA, WEBB JG, PIBAROT P, ALTWEGG L, DUMONT E, THOMPSON C, et al. Comparison of the hemodynamic performance of percutaneous and surgical bioprostheses for the treatment of severe aortic stenosis. J Am Coll Cardiol. 2009;53:1883-91 